\title{
The moderating role of dialecticism in consumer responses to product information $i s$
}

\author{
Haizhong Wang ${ }^{\mathrm{a}, 1}$, Rajeev Batra ${ }^{\mathrm{b}, *, 1}$, Zengxiang Chen ${ }^{\mathrm{c}, *, 1}$ \\ ${ }^{a}$ School of Business, Sun Yat-sen University, 135 Xing Gang Road, Guangzhou 510275, China \\ ${ }^{\mathrm{b}}$ Ross School of Business, University of Michigan, 701 Tappan Street, Ann Arbor, MI 48109-1234, USA \\ ${ }^{\mathrm{c}}$ School of Tourism and Service Management, Nankai University, Tianjing, China
}

Accepted by Durairaj Maheswaran, Editor; Associate Editor, Vanitha Swaminathan

Received 25 September 2013; received in revised form 21 October 2015; accepted 28 October 2015

Available online 5 November 2015

\begin{abstract}
We show that consumers high in dialecticism - the tolerance of contradictions and the expectations of change (Peng \& Nisbett, 1999) - respond differently than low-dialecticism consumers to messages that have purely positive, mixed, or purely negative information. We find first that for low dialectics, felt ambivalence-and discomfort-is greater for mixed information messages than for negative or positive information. For high dialectics, however, mixed information leads to high felt ambivalence, but not to high discomfort. When given univalent positive or negative information messages, high dialectics have more thoughts about information opposite in valence to that presented, when presented with negative information messages. As a result, for high dialectics, univalent negative information produces the same high felt ambivalence, and even greater discomfort, than do mixed information messages. Through these non-parallel effects, we show that the relationship between felt ambivalence and discomfort is itself moderated by dialecticism. Through three experiments and using a novel manipulation of dialecticism, we replicate and generalize these effects and provide process evidence.
\end{abstract}

(C) 2015 Society for Consumer Psychology. Published by Elsevier Inc. All rights reserved.

Keywords: Dialecticism; Information processing; Attitude ambivalence; Discomfort; Cross-cultural psychology; Persuasion

\section{Introduction}

While many research studies have examined the impact on consumer information processing of regulatory focus and self-construal (e.g., Lee, Aaker, \& Gardner, 2000), few have investigated the effects of a more "dialectical" processing style. As Wyer and Hong (2010, p.631) point out, although dialectical processing style is well researched in the cross-cultural literature

is The authors acknowledge the research grants awarded by the National Natural Science Foundation of China (71572207; 71572084; 71172164; 71202164), and the helpful comments of L.J. Shrum and Joseph Priester.

* Corresponding authors.

E-mail addresses: whz@chinabrandcenter.org (H. Wang), rajeevba@umich.edu (R. Batra),chzengx@gmail.com (Z. Chen).

1 The authors contributed equally.
(East Asians typically being higher on dialecticism than North Americans: Nisbett, Peng, Choi, \& Norenzayan, 2001; Peng \& Nisbett, 1999), its role in consumer information processing has not been examined in depth. Dialecticism is a style of information processing that focuses on tolerance of contradictory information, as well as expectations that the environment will continually change (Nisbett et al., 2001; Peng \& Nisbett, 1999). Since dialecticism concerns the expectations of contradictions in everyday life, it ought to be especially useful in understanding how consumers react to product messages that contain mixedvalence information, such as that becoming more influential today through social media and online reviews (e.g., Amazon. com).

Since our focus here is on responses to product-related messages that contain both, or only, negative and positive information, it is natural that we examine the effect of these 
messages on attitude ambivalence, on which there is an extensive prior literature. ${ }^{2}$ Ambivalent attitudes are attitudes that contain independent elements of both positivity and negativity, rather than overall unidimensional evaluations (Kaplan, 1972; Priester \& Petty, 1996, 2001). We also study the effects on downstream discomfort. We contribute to the literature by showing for the first time that while higher dialecticism consumers ("high dialectics") have the same strong degree of attitudinal ambivalence to mixed and univalent negative, but not positive, product information, low dialectics feel more ambivalence to mixed information than to either univalent (positive or negative) messages. Second, we show a very important asymmetry in the effects of univalent positive-only versus negative-only messages. For high dialectics, but not low, negative messages evoke more ambivalence than positive ones. This finding is new, and our theoretical development provides a rationale for why this asymmetry should arise. We make a third contribution by showing that the number of self-generated cognitive "anticipated conflicting responses" (Priester, Petty, \& Park, 2007) underlies these differences in ambivalence.

If consumers feel ambivalent about an attitude object, it seems logical that they might also feel a sense of discomfort, and some prior research has shown this effect (e.g., van Harreveld, van der Pligt, \& Yael, 2009), even equating felt ambivalence with consequent tension and discomfort (e.g., Harmon-Jones, 2000; Priester et al., 2007). We make a fourth contribution by showing that the relationship between attitude ambivalence and discomfort is itself moderated by dialecticism. Though low dialectics show parallel effects across felt ambivalence and discomfort, high dialectics do not. Thus, felt ambivalence and discomfort are two distinct outcomes. We show these effects in a series of experiments that use multiple complementary operationalizations.

\section{Theoretical background and hypotheses}

\section{Dialecticism}

As a universal philosophy focusing on how to deal with contradictions in life (Nisbett et al., 2001; Peng \& Nisbett, 1999), dialecticism is characterized by two elements. The first is the belief that the world (reality) is constantly changing, so that oppositions and paradoxes are continuously being created. Thus the two sides of an apparent contradiction exist in active harmony: bad becomes good, hate becomes love, and virtue becomes evil. Therefore-the second key element-in this changing world, those high in dialecticism believe that contradictions can be regarded as natural, to be accepted and tolerated (Peng \& Nisbett, 1999; Spencer-Rodgers, Peng, \& Wang, 2010). By accepting contradictions, more-dialectical people seek the middle "way" between extreme propositions.

\footnotetext{
${ }^{2}$ Note that we do not study effects on attitude valence, because messages containing positive vs. mixed vs. negative information about a product will lead to obvious and uninteresting effects on attitudes toward the product: positive information should naturally yield the most positive attitudes and negative the least. We do however report these data in the methodological appendix, for completeness.
}

Less-dialectical people tend to pursue a single truth and have a preference for consistency.

We note here that dialecticism has been shown to be different from constructs such as collectivism/individualism (Triandis, 1995), interdependent self-construal (Markus \& Kitayama, 1991), and preference for consistency (Cialdini, Trost, \& Newsom, 1995). It should also be clearly noted that while dialecticism has been established as a thinking style, this does not mean that high dialectics process information in a more heuristic, top-down manner (versus more systematically). On the contrary, research has shown that high dialectics often search for and use more information, in forming judgments, than low dialectics (e.g., Choi, Dalal, Kim-Prieto, \& Park, 2003; Monga \& John, 2008). High dialectics are also more likely to ascribe causality to situational factors and to multiple actors; low dialectics hold individual actors responsible.

Given its importance, dialecticism has been studied in social psychology for many years, e.g., in the literatures on self-esteem (Spencer-Rodgers, Peng, Wang, \& Hou, 2004), cross-cultural psychology (Spencer-Rodgers et al., 2010), and emotional experience (Miyamoto, Uchida, \& Ellsworth, 2010). However, its use is very limited in consumer research. Mick and Fournier (1998) described consumers' responses to new technology as dialectical, both "a blessing and a curse," but did not measure this construct. A partial conceptualization of dialecticism, the propensity to accept duality, was operationalized by Williams and Aaker (2002), but only through cultural background (Anglo or Asian American) and age. This has obvious limitations because it potentially confounds the across-culture difference in duality with other un-controlled differences (Aaker \& Sengupta, 2000, p.70, footnote 3; Williams \& Aaker, 2002, p. 645).

\section{Attitudinal ambivalence}

Many topics in life evoke evaluative reactions that are simultaneously both positive and negative, and thus conflicting (e.g., toward immigration or nuclear energy). We adopt the terminology of Spencer-Rodgers et al. (2004) to define ambivalent attitudes as those that simultaneously contain evaluations of both positive and negative valence (the coexistence of evaluative opposites). This requires the simultaneous accessibility of both the positive and negative components of attitude (Newby-Clark, McGregor, \& Zanna, 2002).

Previous researchers have conceptualized and measured attitudinal ambivalence in two different ways (Priester \& Petty, 1996). Objective (or intrinsic) ambivalence concerns the extent to which the inherent characteristics of the message are capable of evoking separate positive and negative evaluations (e.g., Sengupta \& Johar, 2002, p. 46; Williams \& Aaker, 2002, p. 640). Thus, measures of objective ambivalence assess the positive and negative assessments of the attitude object's properties separately and then combine them, using mathematical models (Kaplan, 1972; Priester \& Petty, 1996; see Appendix). Since objective ambivalence refers to characteristics of the message itself, not experienced feelings, it is relatively unaffected by situational/contextual factors - including the degree to which 
the consumer is high or low in dialecticism. Thus we use it only as a measure of the strength of our information-type manipulations.

The second type of attitudinal ambivalence-felt ambivalence-becomes our logical outcome. This type of ambivalence concerns how much a particular respondent actually experiences contradictory feelings toward the object, because it is perceived to contain contradictory elements in that particular situation (Newby-Clark et al., 2002; Priester \& Petty, 1996; van Harreveld et al., 2009). Priester and Petty (2001) point out that felt ambivalence is affected not just by message characteristics (e.g., objective ambivalence) but also by intrapersonal factors (e.g., conflict between current and desired attitudes) and by interpersonal situational factors (e.g., the discrepancy between one's own attitudes and close others' attitudes). Thus, there are other determinants of felt ambivalence besides the extent of objective ambivalence. Felt ambivalence is measured by asking individuals about the extent to which their felt attitudes toward an attitude object are one-sided or mixed (Priester \& Petty, 1996).

Naturally, when the presented information is itself mixed in nature (such as a user-generated review in online media), both positive and negative attitudinal components should be accessible, creating felt ambivalence. Importantly, however, Priester et al. (2007) demonstrated that even when presented with univalent information that does not evoke any (or few) conflicting reactions right away, some consumers can believe that there might exist product information or attributes, of the opposite valence, of which they are presently unaware-and thus still feel ambivalence, because of "anticipated conflicting reactions" (hereafter, ACRs). Priester et al. (2007), p.12), "many people recognize that there are often two sides to every story and that nothing is perfect (or completely worthless)." They argue that these ACRs should occur mostly when people are unaware of any specific attributes that are opposite to their dominant reactions - so that ACRs should not arise when consumers are explicitly presented with both the positive and negative sides of an argument (as in our mixed information messages, below), but only when consumers are presented with information of only one valence. Therefore, in our studies below, we argue and show that univalent, not just mixed, information can create felt ambivalence.

Priester et al. (2007) conceptualized an ACR as being a thought or belief about the existence of missing information of the opposite valence. They measured ACRs by asking participants, "To what extent do you anticipate that the product has negative (positive) qualities and attributes that you don't know about?"3 While such a thought is a type of cognitive response $(\mathrm{CR})$, it is not one in which a claim in the incoming message is either supported or countered concerning its accuracy. Rather, an ACR focuses on the completeness of information (e.g., do I have enough information about this product?), in particular the completeness of information that is of the valence

\footnotetext{
${ }^{3}$ The specific scales were anchored with zero equal to "do not anticipate any negative (positive) qualities and attributes" and 10 equal to "anticipate many negative (positive) qualities and attributes." For further details about these ACRs, see Priester et al. (2007, p.12).
}

opposite to that of the information actually presented (Rucker, Tormala, Petty, \& Briñol, 2014, p.123). Because they reflect concerns about completeness, not accuracy, ACRs are not indicators of skepticism or (lack of) trustworthiness of the presented information. Rucker et al. (2014) show that such an ACR-type concern about the completeness of the information presented can decrease attitude certainty, while the usual support and counter argumentation, or the source derogation cognitive responses that reflect concerns about trustworthiness, should affect attitude valence. However, while ACRs are thus different from the usual CRs in content, they should still be like CRs in that both require cognitive effort, thus high motivation and ability to process and respond; both are thus more likely and numerous when incoming information is negative rather than positive, provoking more systematic processing overall (see below).

Priester et al. (2007) found that these ACRs mediated the effect of the number of product attributes on felt ambivalence. Earlier data in Priester and Petty (2001) also supports the ambivalence-creating effects of such ACRs evoked by receiving univalent messages. Thus we argue below that the generation of ACRs, not broader overall CR production (or the degree of systematic processing, or involvement) is most related to the level of felt ambivalence.

Felt ambivalence for different types of messages: Low dialectics

For low dialectics responding to mixed vs. univalent messages, the literature suggests two opposing hypotheses. On the one hand, given their definitional low tolerance for contradiction, it could be argued that mixed information will elicit more ambivalent feelings than univalent information, since positive and negative attitudinal components are more accessible jointly in mixed than in univalent information. Prior literature has shown that felt ambivalence increases with the simultaneous accessibility of both positive and negative attitudinal components (Newby-Clark et al., 2002). Empirical evidence exists that low dialectics (Anglo-Americans) feel more ambivalence when facing mixed, than univalent, information (Williams \& Aaker, 2002).

On the other hand, our literature review suggests that positive and negative univalent messages can also create high felt ambivalence for consumers, because the ACRs that create felt ambivalence appear mostly for univalent, and not mixed, messages (Priester et al., 2007, p.12; Rucker et al., 2014). However, this second theoretical expectation comes with a crucial caveat. Priester et al. (2007, p.12) point out that these ACRs that create felt ambivalence are more likely among individuals who "recognize there are often two sides to every story." Low dialectics should thus not be likely to generate ACRs to univalent messages, and then feel ambivalence.

Should this moderation of ACR generation and felt ambivalence by low dialecticism occur equally strongly for both positive and negative univalent messages? Recent literature suggests this should not be so. As much prior research has shown, negative information is usually less ambiguous and more diagnostic, for product judgments, than positive or neutral cues (Herr, Kardes, \& Kim, 1991, p.460; Schwarz \& Bless, 1991, p.61; Skowronski \& Carlston, 1989, p.134); thus, it should receive more processing. 
Negative information also implies a problematic situation, a higher level of risk and danger, and a lower level of safety (Schwarz \& Bless, 1991, p.60); it is thus adaptively better to process and weight negative information more than positive information (e.g., Baumeister, Bratslavsky, Finkenauer, \& Vohs, 2001). These characteristics lead to a reduced reliance on simple heuristics and more detailed processing, when negative (versus positive) information is received. Prior research also shows the asymmetric processing of information when consumers are in positive vs. negative states of mind or affective states, with negative states of mind triggering deeper and more systematic processing (e.g., Schwarz \& Bless, 1991).

Thus, all these streams of literature support the expectation that - as a "main effect" - univalent negative information should trigger more systematic processing than univalent positive information should, leading to more CR production of all types. Thus for low dialectics (as with high dialectics, see below), negative information should evoke more systematic processing (thus more total CR production overall) than positive messages. However, since low dialectics are not expecting a different side to the presented story, these increased CRs for low dialectics should be of the same valence as that of the incoming message information, not ACRs concerning opposite-valence information. The number of ACRs about opposite-valence information for low dialectics should thus be no higher for negative than for positive univalent messages. As a result, felt ambivalence should also not be higher for low dialectics just because the incoming information is negative (vs. positive): it should be at low, roughly equal levels, for both. Thus:

H1a. For low dialectics, mixed information will cause a high degree of felt ambivalence, while both negative and positive information messages will cause a low degree of felt ambivalence (with no difference between them).

\section{Felt ambivalence for different types of messages: High dialectics}

For high dialectics, it might initially seem that since they expect the world to be full of contradictions, they should feel little ambivalence when presented with mixed information messages. However, further analysis shows even high dialectics should find such mixed messages difficult to evaluate and pass judgment on, since they can easily see that it explicitly contains within itself both positive and negative elements-readily apparent (explicit, clear, definite) contradictions, to use the terminology of Peng and Nisbett (1999). Thus mixed information messages should create a high degree of felt ambivalence (a mixed evaluative reaction, with coexisting evaluative opposites) even for high dialectics.

Note that this expected response by high dialectics to mixed information messages is similar to the expectation above for low dialectics. Such equivalence in response by high and low dialectics to obviously mixed information has in fact been shown in prior research on cross-cultural differences in emotional responses to mixed-information stimuli. For example, Miyamoto et al. (2010, study 2) found that both high dialectics (Japanese) and low dialectics (Americans) form the same level of mixed emotions (i.e., feel positive emotion and negative emotions at the same time) when they were asked to recall a mixed situation (i.e., "the day you started classes in college"). Further, Williams and Aaker (2002) also found no evidence that high-dialectical thinkers experienced less mixed emotions than low dialectical thinkers, when they viewed an advertisement designed to elicit mixed ("bittersweet") emotions.

Next, how should high dialectics respond to univalent (purely positive or negative) information? Since high dialectics expect more (than low dialectics do) that information exists of an opposing nature, the CRs they generate for any message should contain many ACRs, concerning the valence opposite to that of the message information. For a negative-only information message, that triggers more systematic processing, high dialectics should thus produce many more ACRs containing self-generated positive thoughts, coexisting with the negative message-generated thoughts. In this case, high dialectics should see a high level of potential contradiction, between the negative-only information clearly stated within the message, and their own self-generated ACRs about the positive information that the message could, but does not, contain. As with the high level of apparent (obvious) contradiction seen in the mixed-information messages, the high level of potential contradiction seen with the negative message should create high felt ambivalence, since both types of contradiction make positive and negative attitudes simultaneously accessible (Newby-Clark et al., 2002). Thus high dialectics should have high levels of ACRs and felt ambivalence for negative information as well, not just mixed.

For positive information messages, high dialectics should again be likely to have many ACRs involving thoughts of the opposite (i.e., negative) valence, given their more-questioning nature. However, the total number of these ACRs about opposite-valence information should still be relatively low, since the overall level of systematic processing is lower for positive messages than for negative ones, even for high dialectics, per the previously cited research on total systematic processing levels. Thus, felt ambivalence should still be lower for them than for negative information. Thus

H1b. For high dialectics, both mixed and negative information messages will cause a high degree of felt ambivalence (with no difference between them), while positive information messages will lead to a low degree of felt ambivalence.

Since ACR generation, our theoretically justified process that creates these levels of felt ambivalence, should occur mostly when consumers see univalent positive or negative (but not mixed) information (Priester et al., 2007, p.12), our theoretical arguments also imply:

H2a. For low dialectics, both negative and positive information messages will cause a low number of ACRs about oppositevalence information (with no difference between them).

H2b. For high dialectics, negative information messages will cause a high number of ACRs, while positive information messages will lead to a low number of such ACRs. 
H3. The number of ACRs will mediate the influence of univalent information (positive vs. negative) on felt ambivalence, but only for high-dialectical consumers, not low.

\section{Discomfort}

We now develop our hypotheses for discomfort. It has long been assumed that resolution of inconsistency is the natural goal for all consumers, because attitudinal ambivalence is associated with psychic tension (Festinger, 1957). Consumer research has, therefore, tended to equate felt ambivalence with feelings of discomfort - a state where one feels uncomfortable, tense, etc. Harmon-Jones (2000) describes discomfort as a negative feeling of being uncomfortable, sad, anxious, uneasy, and bothered. Consistent with this conceptualization, Williams and Aaker (2002) measured feelings of discomfort using three items of conflicted, confused, and uncomfortable. However, because of the assumed overlap of discomfort with felt ambivalence, the first two items they used are also present in the earlier five-item scale of felt ambivalence in Priester and Petty's (1996); Priester \& Petty, 2001). Similarly, Priester et al. (2007) use a five-item attitude ambivalence scale that combines items for felt ambivalence with an item measuring felt tension.

We argue here, however, that felt ambivalence should be treated as a construct that is distinct from discomfort, because the relationship between the two should itself vary with the consumer's level of dialecticism. Felt ambivalence describes the feeling of difficulty in making an overall evaluation or decision because of the presence or absence of conflicting or contradictory information in the attitude object. Therefore (as in H1a), felt ambivalence ought to be greater when the consumer is faced with mixed (versus univalent) information about the attitude object. But discomfort is a distinctly separate state, going beyond current or anticipated decision difficulty: it is a negative feeling of being uncomfortable, sad, anxious, uneasy, and bothered (Harmon-Jones, 2000). Evidence for this distinction can be found in a study by van Harreveld et al. (2009), in which inconsistent stimulus information always led to felt ambivalence but was related to physiological discomfort only when a choice (for or against the attitudinal issue) actually had to be made. Sengupta and Johar (2002) add to the argument for separation by showing that consumers vary in the felt need to resolve inconsistent attitudes: not all consumers who feel ambivalence need also feel discomfort.

Therefore, in contrast to the equivalence assumed between them in the prior literature, we propose that the relation between ambivalence and discomfort itself ought to vary with the level of dialecticism. Peng and Nisbett (1999, p.750) report that among (low dialectical) Americans, "there is a strong desire to generate counter arguments against a position that one doubts, in order to find a more secure basis for belief in some other position" (emphasis added). They point out that this strong desire is much reduced in (high dialectical) Asian cultures, which are more expecting of apparently contradictory facts. Further, Nisbett et al. (2001) report that the Americans "felt substantial pressure to resolve the contradiction" presented to them (302) while "the Chinese developed a dialectic ... which involves reconciling, transcending or even accepting apparent contradictions" (294, emphasis added).

Given this prior theorizing and evidence, we expect that low-dialectics (e.g., Westerners) receiving a mixed-information message should feel a heightened motivation to resolve the inconsistency or contradiction seen in that mixed message, and thus experience more "strong desire" and "substantial pressure" to resolve the contradictions they see in it (than high dialectics). Thus, a mixed-information message should create a high level of consequent tension and discomfort among low dialectics. Other studies also document that low dialectics feel a high level of discomfort in response to mixed information (e.g., Williams \& Aaker, 2002). When facing univalent (positive- or negative-only) information, since they do not see anything troubling with a one-sided world, low dialectics should not be motivated to generate ACRs in response, or feel pressured to resolve and explain what they are seeing. As a result, low dialectics should not only experience less felt ambivalence but also feel less discomfort. Thus:

H4a. For low dialectics, mixed-information messages will cause a high degree of discomfort, while negative and positive information messages will both cause a low degree of discomfort (with no difference between them).

We now examine what should happen, in terms of discomfort, for higher dialecticism consumers when they face different types of messages. For mixed-information messages, recall our statement earlier that such a message explicitly includes both positive and negative elements, thus visibly containing within itself what Peng and Nisbett (1999) called apparent contradiction. For such mixed-information messages, the self-apparent nature of the contradiction matches what high dialectics expect to find around them. High dialectics - for whom such a mixed state of the world should appear reasonable and normal - should thus be highly likely to accept and tolerate the contradictory facts contained in the message (e.g., Peng \& Nisbett, 1999; Spencer-Rodgers et al., 2004), so that such mixed-information messages should not create high desire- or pressure-to-resolve in high dialectics, and therefore not create high tension and discomfort. Supporting this line of argument, Choi and Nisbett (2000) have shown that Korean participants (high dialectics) display less surprise and greater hindsight bias than American participants (low dialectics) do, when a target's behavior clearly contradicts expectations.

What should be the levels of discomfort felt by high dialectics for univalent information messages? For positive information, we argued in $\mathrm{H} 1 \mathrm{~b}$ and $\mathrm{H} 2 \mathrm{~b}$ that high dialectics should be less likely to generate ACRs, thus only creating low levels of felt ambivalence. Since the level of felt ambivalence is at low levels, high dialectics should also experience just a low need to resolve the felt ambivalence, and thus not experience a high level of discomfort either.

For messages with purely negative information, however, high dialectics are likely to have generated a high level of ACRs (H2b) and thus feel more ambivalence (H1b). Importantly, when these high dialectics get negative-only information, the ACRs 
they self-generate about possible, omitted positive-valence information should be accompanied by greater felt uncertainty, than would be felt from positive-valence arguments explicitly contained within a mixed-information message. That is, in the mixed-message case, both the negative and positive arguments are explicit within the message itself, presenting a self-apparent and definite contradiction, thus evoking low uncertainty. However, in the negative-only message case, the message-presented negative information is explicit and certain, while the self-generated positive thoughts (via the ACRs) are more conjectural and uncertain, creating what we earlier called potential (but not for-sure) contradiction. High dialectics should therefore experience more unease and discomfort from a negative-only message than from a mixed-information message - even though their level of felt ambivalence should be the same across these two types of messages (see H1b). ${ }^{4}$ Thus:

H4b. For high dialectics, negative information messages will cause a high degree of discomfort, while mixed and positive information messages will both lead to a low degree of discomfort (with no difference between them).

While our hypotheses above concerning the differential responses by high dialectics to positive-only versus negativeonly messages have been developed on the basis of substantial theory and prior empirical results, we acknowledge that alternative possibilities exist, especially with regard to underlying processes. Hence we will explore multiple process possibilities in our second study, and explore non-hypothesized outcomes in our testing.

\section{Mediation of discomfort effects by felt ambivalence}

When we combine $\mathrm{H} 1$ with $\mathrm{H} 4$, we can see that for low dialectics, the influence of message information type on felt ambivalence, and on discomfort, shows a consistently parallel pattern, with mixed information causing both greater felt ambivalence and greater discomfort than positive or negative.

\footnotetext{
${ }^{4}$ It might be asked why the potential contradiction in the negative-only message (via ACRs) works differently than the explicit contradiction in the mixed message, such that for high dialectics the negative-only message creates more discomfort but not more felt ambivalence. As argued earlier, felt ambivalence is caused by the simultaneous accessibility of positive and negative thoughts (Newby-Clark et al., 2002), while discomfort is caused by the pressure to resolve apparent contradictions (Peng \& Nisbett, 1999). Thus while felt ambivalence is related to information accessibility, discomfort is related to information certainty. Prior research has shown (Petrocelli, Tormala, \& Rucker, 2007) that attitude certainty is distinct from (though related to) attitude accessibility (p. 31). Therefore, for high dialectics, for both mixed and negativeonly messages, both positive and negative information are simultaneously accessible (in the case of negative-only messages, this happens via the ACRs; for mixed messages, via the explicit information presented), creating high felt ambivalence in both. But the level of uncertainty, and thus discomfort, is higher in the negative information message than in the mixed message: the oppositevalence ACR-generated thoughts in the former are uncertain, while the explicitly-presented information in the latter is apparent/explicit and thus certain.
}

This means that, for low dialectics, the effect of information type on discomfort should be mediated by felt ambivalence, driven by the comparisons between mixed versus negative, and mixed versus positive, but not between negative and positive information.

The expected pattern of results is different for high dialectics. For them, positive information should not create higher felt ambivalence, but both mixed and negative information should. In terms of discomfort, positive information should not create high discomfort, nor should mixed. Discomfort should, however, be high for these high dialectics from negative information. As a result, the mediating role of felt ambivalence should only emerge for them in the comparison between negative and positive, and not in the comparisons between mixed and negative, or mixed and positive conditions. Prior research does not expect such moderated mediation (Hayes, 2013), because it equates the effects of information type on felt ambivalence with those on discomfort and does not expect moderation of these by dialecticism. Formally:

H5. There exists a moderated mediation effect, with felt ambivalence mediating the link from product information type to discomfort, and with dialecticism moderating this mediation process. For low dialectics, this mediating effect is driven by the comparisons between the mixed versus univalent, but not between negative and positive conditions; for high dialectics, this mediating effect is driven by the comparisons between the negative and positive condition, and not in the comparisons between the mixed and univalent conditions.

\section{Overview of experiments}

We will test our hypotheses through three studies. In the first study, we will manipulate dialecticism and examine its moderating role in the relationship of information type, felt ambivalence, and discomfort. Study 2, limiting itself to a comparison of positive versus negative univalent messages, tests the proposed process mechanism of ACRs and also rules out several plausible alternative process explanations. Different from the first two studies that present participants with specific attribute-based product information, our third study presents them with summary product quality information (customer satisfaction ratings) and examines whether the conclusions from the first two studies can be generalized to this context.

\section{Experiment 1}

\section{Design and procedure}

This experiment used a 3 (information type: positive vs. mixed vs. negative) $\times 2$ (dialecticism: high vs. low) between-subjects design. Participants were told that a new MP3 player was going to be launched into the market soon, and its manufacturer was investigating usage patterns and attitudes of university students, who were their target customers. 158 Chinese university students participated, for nominal monetary compensation (\$2.00).

The main experiment had three parts. First, we used a method that combined proverb completion and paragraph-length stories 
to manipulate dialecticism. Five high- or low-dialectical Chinese proverbs were presented at the top of the first page of priming materials (high dialectical example: "Sorrow is born of excessive joy"; low dialectical example: "As you sow, so shall you reap"). Participants were required to fill in one missing word in each proverb and then in the bottom line write down only one common meaning that all five proverbs seemed to share. The second page of priming materials used a paragraph-length story recall technique. Here, the second half of the first sentence in the paragraph used the expression "as the proverb ' $x$ ' implies" to strengthen the association between the two tasks of proverb completion and the paragraph, and to increase the strength of the priming manipulation. The high-dialectical priming materials were intended to activate greater tolerance of contradictions and to create thoughts of two-sided resolution (i.e., there is no right answer); the low-dialectical priming materials were intended to activate the law of non-contradiction (i.e., there was a very clear and single answer). We used an independent pretest $(n=45$, male $=20)$ to validate the manipulation (details of these materials and the pretest are in the methodological appendix).

Second, we manipulated the stimulus product attribute information to be either purely positive, purely negative, or mixed. The four attributes of the MP3 player on which information was provided (randomly rotated) were tone quality, hours of use, storage capability, and degree of operational convenience. The positive information condition showed that all four attributes were rated five stars and had positive descriptions of all four attributes. The negative information was rated at the one-star level. In the mixed-information condition, two attributes of Yiven were rated five stars and the other two rated one star, such that the number of positive and negative attributes were equalized (Sengupta \& Johar, 2002). After reading the manipulated description, participants were asked questions about the dependent variables of felt ambivalence and discomfort (objective ambivalence was measured as a manipulation check).

\section{Measures}

Objective ambivalence was measured by six 4-point scales about the stimulus product (Kaplan, 1972): "favorable/ unfavorable," "positive/negative," and "beneficial/harmful" $(\alpha=.83$ and .90 for the favorable and unfavorable stimuli). The values of objective ambivalence computed via the SIM, CRM, and GTM models (see appendix) yielded similar results, and only the SIM results are reported for brevity. For felt ambivalence, participants used three statements to report their feelings about the stimulus product $(1=$ not at all conflicted/not at all mixed/not at all indecisive, $7=$ very conflicted/very mixed/ very indecisive; $\alpha=.72$ ), following Priester and Petty (1996). Discomfort was measured via three items, uncomfortable/uneasy/ bothered (Harmon-Jones, 2000), on a 5-point scale ( 1 = not at all felt, $5=$ strongly felt; $\alpha=.81$ ). In this experiment (as in the later 2), the multi-item scales for felt ambivalence and discomfort correlated moderately $(.32, p<.01)$, and clearly loaded on different factors in an EFA.
Results

\section{Manipulation checks}

In addition to conducting the pretest (see appendix), we also used other indicators to examine whether our dialecticism manipulation was working as intended. (Details appear in the Appendix, adjacent to the description of the manipulation.) These tests showed that the proverbs we were using were appropriate and familiar to our participants and were equally trustworthy across conditions. Thus, our proverb completion task appears to be a valid and non-confounded priming technique. The manipulation of product attribute information type was also successful. Results revealed that there was only a main effect of information type on objective ambivalence $(F(2,152)=21.31, p<.001)$, with mixed-information exposure causing a higher level $(M=1.98$, by the SIM model) than positive $(M=1.05 ; t(155)=-6.40, p<.001)$ and negative $(M=1.23 ; t(155)=-5.07, p<.001)$. There was no significant difference across the positive vs. negative conditions $(t(155)=-1.28, p>.10)$.

\section{Felt ambivalence}

There was a significant interaction of information type by dialecticism $(F(2,152)=6.11, p<.01)$. Contrast comparisons revealed that for low dialectics, mixed information evoked significantly higher levels of felt ambivalence $(M=4.39)$ than negative $(M=2.81 ; F(1,152)=22.05, p<.001)$ as well as positive $(M=3.08 ; F(1,152)=13.58, p<.001)$. Further, there was no significant difference between the positive and negative conditions $(F 1,152)<1, p>.10)$. Thus, H1a was supported. However, for high dialectics, mixed information was not significantly different than negative $\left(M_{\text {mixed }}=4.01, M_{\text {negative }}=\right.$ $3.96 ; F(1,152)<1, p>.10)$. Mixed information caused greater felt ambivalence than positive $(M=3.03 ; F(1152)=10.57$, $p<.001)$, and negative information evoked greater felt ambivalence than positive $(F(1152)=9.03, p<.01)$. H1b is supported.

\section{Discomfort}

Analysis showed that there existed a significant interaction effect of information type by dialecticism $(F(2,152)=4.58$, $p<.05$ ). Contrast analysis revealed that for low dialectics, mixed information $(M=2.80)$ created significantly more discomfort than both positive $(M=2.06 ; F(1,152)=10.70$, $p<.001)$ and negative $(M=2.25, F(1,152)=6.42, p<.05)$. There was no significant difference between positive and negative information $(F(1,152)<1, p>.05)$, supporting H4a. For high dialectics, there was no significant difference in discomfort between mixed $(M=2.34)$ and positive information $(M=2.15 ; F(1,152)=1.04, p>.05)$. But negative information caused more discomfort than did positive $\left(M_{\text {negative }}=2.68\right.$, $\left.M_{\text {positive }}=2.15 ; F(1,125)=7.47, p<.01\right)$ and marginally more discomfort than mixed $\left(M_{\text {mixed }}=2.34 ; F(1152)=2.82\right.$, $p=.09$ ). Therefore, $\mathrm{H} 4 \mathrm{~b}$ finds support, but only at $p<.10$.

\section{Moderated mediation}

We used a bias-corrected bootstrapping procedure with a multi-categorical independent variable (5000 samples; Hayes, 
2013, model 4) to test the process of information type $\rightarrow$ felt ambivalence $\rightarrow$ discomfort, separately for higher and lower dialecticism. For low dialectics, we had predicted that the mediation of felt ambivalence only exists when comparing positive versus mixed, and negative versus mixed conditions. So we created two dummy variables to capture the three experimental conditions, with mixed information as the baseline condition. The analysis showed that the effect of information type on discomfort was mediated by felt ambivalence $(95 \% ; \beta=-.26$, CI excluding zero, from -.62 to -.03) in the comparison between the positive and mixed-information conditions, as also in the comparison between negative and mixed conditions (95\%; $\beta=-.31$, CI excluding zero, from -.73 to -.02$)$. Furthermore, the direct effects of information type on discomfort in these two comparisons were not significantly different (respectively, $\beta=-.47$, CI including zero, from -.99 to $.04 ; \beta=-.23$, CI including zero, from -.75 to .28 ), indicating the presence of indirect-only mediation. Positive and negative messages evoked the same level of felt ambivalence and discomfort, making mediation tests unnecessary.

For high dialectics, we expect mediation by felt ambivalence only when comparing positive and negative information. The same bootstrapping analysis (5000 samples; Hayes, 2013, model 4) revealed mediation when comparing these conditions $(95 \% ; \beta=.11$, CI excluding zero, from .01 to .29). The direct effect was significant $(95 \% ; \beta=.42$, CI excluding zero, from .04 to .80$)$, indicating complementary mediation. Since high dialectics formed the same level of felt ambivalence in negative and mixed conditions, and the same level of discomfort in positive and mixed conditions, mediation tests across these conditions were unnecessary. H5 finds support.

\section{Discussion}

This first experiment shows that for low dialectics, mixed information causes greater felt ambivalence and discomfort than univalent negative or positive information, in line with prior research (Priester \& Petty, 1996, 2001; Sengupta \& Johar, 2002; Williams \& Aaker, 2002). But it also extends prior research by additionally showing that for high dialectics, negative-only information causes the same high level of felt ambivalence, and even more discomfort, than mixed information (with positive information having both least ambivalence and discomfort). As well, the experiment demonstrates the dissociation between felt ambivalence and discomfort for high dialectics. It then provides evidence for moderated mediation.

Two issues remain after this first experiment. First, while the results support our outcome expectations of $\mathrm{H} 1, \mathrm{H} 4$, and $\mathrm{H} 5$, they do not provide any process evidence that it is ACRs that are creating the felt ambivalence in the univalent information conditions, as our theory development above (H2, H3) argued. Second, the prior study does not rule out some alternative process explanations that might explain the asymmetrical effects between the positive and negative information conditions. Are high dialectics simply more skeptical about the presented negative information, leading to more uncertainty about its validity (and more conflicting reactions), thus creating more felt ambivalence? Were the high dialectics-more expecting of change and more tolerant of contradictions - simply more optimistic than the lower dialecticism consumers, thus evoking more conflicting positive thoughts to the negative information, creating more felt ambivalence? Were high dialectics simply processing the negative messages with greater effort and involvement? In order to test these alternative explanations, the next study measured participants' trust in the product descriptions, their optimism, and their involvement. Since the more interesting results of the first study are those involving the negative vs. positive univalent conditions, the next study limited itself to these two information conditions.

\section{Experiment 2}

\section{Design and procedure}

This experiment used a 2 (manipulated dialecticism: high vs. low) $\times 2$ (information type: positive vs. negative) between-subject design. 105 undergraduate Chinese students were recruited (paid $\$ 2.00$ ) and randomly assigned into four groups. The procedure and materials were similar to experiment 1 . A new product category (electronic tablet "Pads") was used. Participants firstly completed the same manipulation of dialecticism as in Study 1, then saw a Pad's description (four pretested attributes: storage capability, running speed, weight, and the degree of friendly interface) and finally completed the desired measures. Participants first provided their response to three valence measures $(1=\mathrm{bad} /$ negative/unfavorable, $7=$ good/positive/favorable, $\alpha=.97)$, used as the information valence manipulation check, and then provided measures of felt ambivalence $(\alpha=.64)$ and discomfort $(\alpha=.89)$.

We measured anticipated conflicting responses (ACRs) through a rating item: for the negative (positive)-information condition, "Did you suspect that there was some positive (negative) information about this Pad that was not presented in the message?" ( 1 = never thought that, 7 = fully thought that $)$. This single item is very similar to the single item used by Priester et al. (2007): "To what extent do you anticipate that the (brand) has some positive (negative) qualities and attributes that you don't know about?" Finally, respondents answered questions about involvement in the task (two items, $r=.57$ ), the extent to which they trusted the information in the stimuli (three items, $\alpha=.92$ ), and their levels of optimism at that time (six items, $\alpha=$ $.54)$. Details appear in the methodological appendix.

\section{Results}

\section{Manipulation checks}

We used the valence of responses to the stimulus information as our message information valence manipulation check. There was only a significant main effect of information valence: respondents rated the positive information message more favorably $(M=5.49)$ than they did the negative $(M=3.14$; $F(1,101)=115.07, p<.001)$. Since our dialecticism manipulation had already been successfully pretested and used, and 
since the dialecticism scale required using a long list of 14 items, we elected to not use that scale within this study.

\section{ACRs}

First, there was a main effect of information type $(F(1,101)=$ $8.76, p<.01)$, with consumers reporting larger numbers on the ACR rating scale when they faced negative information $(M=4.96)$ than positive $(M=3.69)$. This is consistent with the voluminous prior research, cited earlier in our theoretical development, showing more systematic processing overall in response to negative (versus positive) information. Second, this main effect was moderated by dialecticism $(F(1,101)=3.24$, $p=.075)$. As expected $(\mathrm{H} 2 \mathrm{a})$, low dialectics reported the same relatively lower level of ACRs when given either negative or positive information $\left(M_{\text {negative }}=4.42\right.$ vs. $M_{\text {positive }}=3.93, F(1$, $101)<1, p>.40$ ). In contrast (supporting H2b), high dialectics had a higher value on the ACR reporting scale in the negative than in the positive condition $(5.48$ vs. $3.44, F(1,101)=11.35$, $p<.01)$. From another perspective, in the negative information condition, high dialectics had higher levels on the ACR scale (5.48) than low dialectics $(4.42, F(1,101)=3.22, p=.076)$, while in contrast, there was no significant difference in the positive information condition $\left(M_{\text {higher dia. }}=3.44\right.$ vs. $M_{\text {lower dia. }}=3.93$, $F(1,101)<1, p>.47)$. See Fig. 1 .

\section{Felt ambivalence}

Replicating earlier results, there was a significant interaction effect of information type by dialecticism $(F(1101)=8.25$, $p<.01)$. Further analyses revealed that for high dialectics, negative information evoked more felt ambivalence $(M=3.80)$ than positive $(M=2.95 ; F(1,101)=6.34, p=0.013)$. In contrast, for low dialectics, there was no significant difference of felt ambivalence between the positive and negative information conditions $\left(M_{\text {positive }}=3.32\right.$ vs. $M_{\text {negative }}=2.78$; $F(1,101)=2.42, p>.12)$.

\section{Discomfort}

The interaction effect of information exposure and dialecticism was significant $(F(1,101)=5.49, p=0.02)$. Simple effect tests revealed that for high dialectics, negative information did lead to more $(M=2.65)$ discomfort than positive $(M=1.80 ; F(1101)=$ $12.53, p=0.001)$. For low dialectics, there was no significant difference in discomfort between these two conditions $\left(M_{\text {positive }}=1.93\right.$ vs. $\left.M_{\text {negative }}=1.97 ; F(1101)<1, p=.83\right)$.

\section{Process testing: ACRs to felt ambivalence}

To test the mediating role of ACRs on felt ambivalence (H3), we used a bias-corrected bootstrapping method (5000 samples; Hayes, 2013, model 7). For high dialectics, we found that the ACRs mediated the effect of information type on felt ambivalence $(\beta=-.24,95 \% \mathrm{CI}=-.62$ to -.035$)$. Further, the direct effect of information type on felt ambivalence was not significant $(\beta=-.0088,95 \% \mathrm{CI}=-.52$ to .50$)$, indicating indirect-only mediation. In contrast, such mediation by ACRs was not supported for low dialectics $(\beta=-.06,95 \% \mathrm{CI}=-.32$ to .06). Thus, our process data on ACRs support our theory-based expectations for felt ambivalence (H3).

\section{Moderated mediation}

Since ACRs were proposed earlier as the reason why high-but not low-dialectics felt more ambivalence, and consequently more discomfort, we next tested the complete process model (information type $\rightarrow$ ACRs $\rightarrow$ felt ambivalence $\rightarrow$ discomfort) using the bootstrapping method (5000 samples; Hayes, 2013, model 6). We predicted that the proposed process sequence would only find support among high dialectics. As Fig. 2 shows, the effect of information type on discomfort was indeed mediated by ACRs and felt ambivalence $(95 \% ; \beta=-$ .07 , CI excluding zero, from -.30 to -.004$)$ for high dialectics, with the direct effect being significant $(\beta=-.77$, CI excluding zero, from -1.29 to -.24 ), indicating complementary mediation. However, as expected, this process model did not find support among low dialectics $(95 \% ; \beta=-.003$, CI including zero, from -.05 to .01 ), supporting H5.

\section{Ruling out alternative accounts}

One alternative explanation for our experimental results could be that high dialectics were simply more distrusting about our focal product's negative-only information description, leading to more uncertainty and doubt about the product's qualities, causing higher felt ambivalence and discomfort. However, two additional mediation analyses showed that for high dialectics, after controlling for the level of message trustworthiness via our measure, ACRs still mediated the effect of information type on felt ambivalence and discomfort. Nor did our trustworthiness measure itself mediate the effect of information type on felt ambivalence. We also tested whether high dialectics-more expecting of change and more tolerant of contradictions-were simply more optimistic than low dialectics, thus evoking more conflicting positive thoughts when presented with the negative-only information, leading to more felt ambivalence. Mediation tests of the process of information type $\rightarrow$ optimism $\rightarrow$ felt ambivalence showed that in neither high nor low dialectics are these mediating optimism processes significant.

A third rival explanation could be that high dialectics - who should expect more, and thus search more, for conflicting information-simply processed the negative message with more involvement and effort, thus producing more ACRs, leading to more felt ambivalence. We had argued that it was ACRs in particular, and not the overall level of systematic processing, or involvement, that led to felt ambivalence. In our data, we did find higher involvement levels when greater systematic processing might be expected overall: high dialectics have a marginally higher involvement in negative than in positive information conditions, while low dialectics reported the same and relatively lower level of involvement in both negative and positive information conditions. We then tested the possibility that involvement mediated the effects of information valence on the number of ACRs produced, and then on the level of felt ambivalence, for high dialectics. However, these mediation tests showed that for high dialectics, the process of information type $\rightarrow$ involvement $\rightarrow$ ACRs $\rightarrow$ felt ambivalence is not significant. Details of all these tests are omitted here for brevity but are available upon request. In short, all these additional analyses were supportive of our theoretical 
EXPERIMENT 2: EFFECT OF DIALECTICISM AND INFORMATION TYPE ON ACRs, FELT AMBIVALENCE, DISCOMFORT
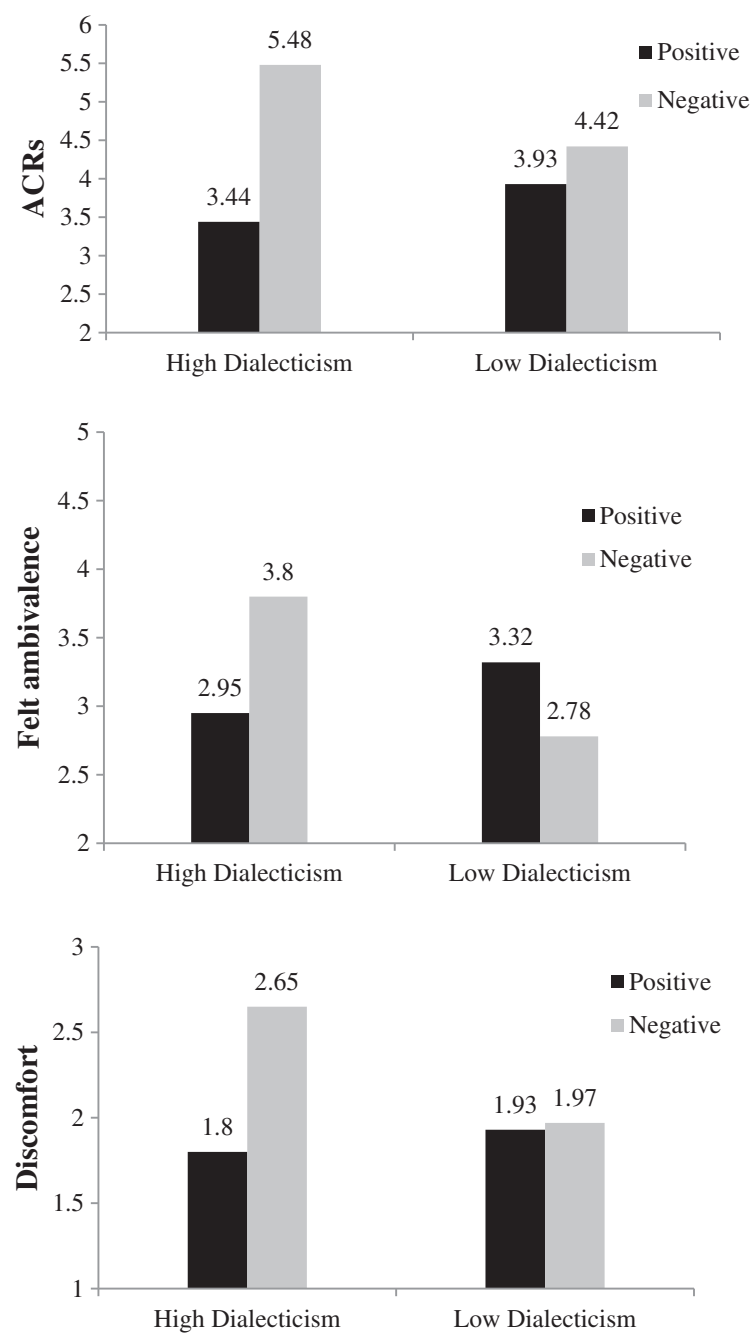

Fig. 1. Experiment 2: Effect of dialecticism and information type on ACRs, felt ambivalence, discomfort. framework, that ACRs drove the asymmetric effect of negative versus positive information, among high dialectics, in creating higher felt ambivalence and discomfort.

\section{Discussion}

The results of this second study make several additional contributions. Beyond replicating the earlier effects on felt ambivalence and discomfort in a new product category, they provide support for the proposed underlying processes, in two ways. First, our measure here of consumers' ACRs provided supporting evidence for our proposed mechanism. Second, our measures here of consumers' level of trust in the presented information, and their levels of optimism and task involvement, allowed us to rule out some possible alternative process explanations, further increasing our confidence in our underlying theoretical framework.

Some further issues remain, addressed below in our final Study 3. The stimuli employed in the two prior experiments for information type used specific, rather than summary, attribute information. Though such a manipulation of information has become a tradition in the attitudinal ambivalence and related research traditions (Sengupta \& Johar, 2002), there is also widespread use of summary or overall information (such as ratings by Consumer Reports, J.D. Power, etc.) by consumers, so we used such ratings here. Second, within these stimuli, we manipulated information inconsistency (mixed vs. univalent information) by presenting a conflicting (versus uniform) distribution pattern of these other consumers' summary satisfaction judgments.

\section{Experiment 3}

Design and procedure

With 155 undergraduate students from a major Chinese university participating in this experiment, we again used a 3 (information type: positive versus mixed versus negative) $\times 2$

\section{EXPERIMENT 2:THE MEDIATING PROCESSFOR HIGHERDIALECTICISM CONSUMERS}

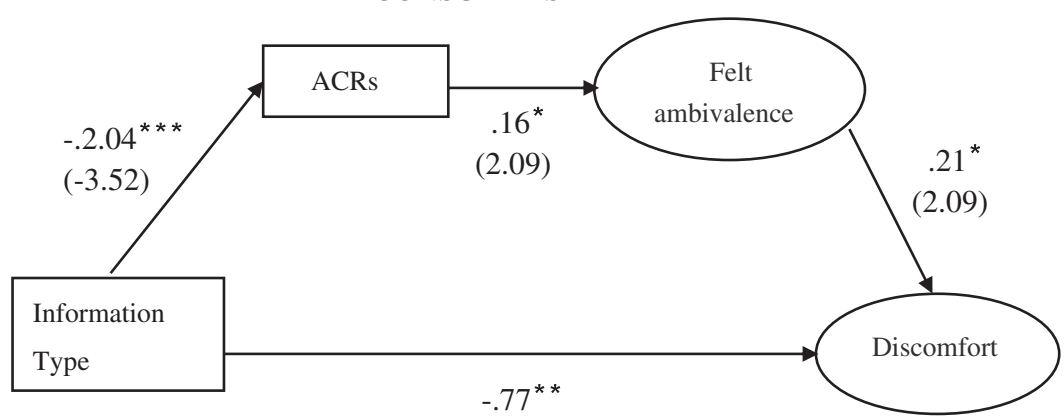

$(-2.93)$

Note: t-values are given in parentheses. Asterisked values are unstandardized coefficients.

Fig. 2. Experiment 2: The mediating process for higher dialecticism consumers. 
(dialecticism: high versus low) between-subjects design. We told participants that a new MP3 player brand, Yiven, was going to be launched into the market soon, for which the company was conducting research. The experimental procedures were similar to those of Experiment 1.

The priming technique and materials for dialecticism were similar to those for Experiment 1, but the stimulus manipulation of information type was different (see methodological appendix). The participants were told that after Yiven was put into the market, an international market research agency was hired by the manufacturer to investigate user satisfaction levels. They were told that customer satisfaction ratings had proven to be a very effective summary measure, resulting from comparisons between consumer expectations and their actual product experience, on multiple attributes. Such information was then presented, as percentage distributions of user ratings on overall satisfaction, from one star ("very disappointed") to five stars ("very satisfied").

In the mixed (inconsistent) information condition, the percentage distribution on the five different levels of satisfaction shown was $10 \%, 25 \%, 30 \%, 25 \%$, and $10 \%$ (very disappointed/somewhat disappointed/neither satisfied nor disappointed/somewhat satisfied/ very satisfied). The mean value of customer satisfaction in this mixed condition was three stars (neither satisfied nor disappointed). In the negative information condition, customer satisfaction covered only the lower levels from one star (very disappointed) to three stars (neither satisfied nor disappointed): the presented distribution was $30 \%, 40 \%, 30 \%, 0 \%$, and $0 \%$. The mean value in this negative condition was two stars (somewhat disappointed). In the positive information condition, the presented distribution was $0 \%, 0 \%, 30 \%, 40 \%$, and $30 \%$. The mean value of customer satisfaction in this positive information condition was four stars (somewhat satisfied).

The prior scales were used for objective ambivalence $(\alpha$ for three positive and three negative questions $=.86$ and .89 , respectively): felt ambivalence $(\alpha=.65)$ and discomfort $(\alpha=.87)$.

Results

\section{Manipulation checks}

We used the short version of the DSS (dialectical self scale, Spencer-Rodgers et al., 2010) as our dialecticism manipulation check $(\alpha=.66)$. ANOVA showed that participants in the high dialecticism condition scored higher $(M=4.95)$ than those in the low $(M=4.51 ; F(1,153)=7.46, p<.05)$. The manipulation of product attribute information type was also successful. Results revealed that there was only a main effect of information type on objective ambivalence $(F(2,149)=10.28, p<.001)$, higher for mixed $(M=1.86)$ than positive $(M=1.30 ; t(152)=4.59$, $p<.001)$ and negative $(M=1.49 ; t(152)=2.81, p<.01)$. There was no significant difference in the objective ambivalence ratings of the positive versus negative conditions $(t(152)=1.61$, $p>.10)$.

\section{Felt ambivalence}

Replicating earlier results, we found a significant interaction effect of information type by dialecticism $(F(2,149)=3.28$, $p<.05)$. For low dialectics, mixed information $(M=3.97)$ evoked higher felt ambivalence than did both positive ( $M=$ $2.91 ; F(1,149)=13.48, p<.001)$ and negative $(M=2.95$; $F(1,149)=10.62, p<.001)$. However, there was no significant difference between positive and negative $(F(1,149)<1$, $p>.10$ ). H1a again finds support. As hypothesized, the pattern was different for high dialectics. Contrast analysis showed that mixed information $(M=3.83)$ evoked the same high level as negative $(M=3.92 ; F(1149)<1, p>.10)$. Both mixed $(F(1,149)=6.77, p<.01)$ and negative information $(F(1$, $149)=7.29, p<.01)$ created significantly higher levels than positive $(M=3.0)$, supporting H1b.

\section{Discomfort}

We again found a significant interaction effect of information type by dialecticism $(F(2,149)=4.73, p<.01)$. Further analysis showed that for low dialectics, mixed information evoked significantly more discomfort $(M=3.07)$ than both positive $(M=2.17 ; F(1,149)=12.58, p<.001)$ and negative $(M=2.50 ; F(1,149)=4.52, p<.05)$ information. Further, there was no significant difference in the extent of discomfort caused by positive versus negative information $(F(1,149)=$ $1.79, p>.10)$. Thus, $\mathrm{H} 4 \mathrm{a}$ was again supported.

For high dialectics, however, the influence pattern differed greatly. Specifically, it was negative (rather than mixed) information $(M=3.03)$ that evoked the highest levels of discomfort, significantly more than both positive $(M=2.42$; $F(1,149)=4.86, p<.05)$ and mixed $(M=2.46 ; F(1,149)=$ $4.02, p<.05)$. Further, no significant differences in discomfort emerged between mixed and positive information $(F(1,149)<1$, $p>.10$ ), meaning that mixed information evoked almost the same low degree of discomfort as positive information. $\mathrm{H} 4 \mathrm{~b}$ again finds support.

\section{Moderated mediation}

The same bootstrapping test procedures that were applied in Experiments 1 and 2 were used. We obtained similar results, again supporting H5. For low dialectics, the mediating role of felt ambivalence existed only in comparisons between the positive and mixed condition $(95 \% ; \beta=-.23$, CI excluding zero, from -.55 to -.05$)$, and between negative and mixed $(95 \% ; \beta=-.23$, CI excluding zero, from -.56 to -.034$)$. While the direct effect of the first mediation process was significant ( $\beta=-.67$, CI excluding zero, from -1.21 to -.12 ), indicating complementary mediation, the latter mediation process' direct effect was not significant $(\beta=-.35$, $\mathrm{CI}$ excluding zero, from .91 to .22), indicating indirect-only mediation. For high dialectics, the mediation effect of felt ambivalence was only obtained in the comparison between positive and negative information $(95 \% ; \beta=.17$, CI excluding zero, from .0035 to .48 ), and the direct effect of information type on discomfort was not significant $(\beta=.45$, CI including zero, from -.11 to 1.00 ), indicating indirect-only mediation.

\section{Discussion}

Because any experimental test using a limited number of specific product attributes always runs the risk of omitting some 
attributes that contain diagnostic information for individual users, our third experiment used overall summary information; the results were still consistent with those derived from our earlier studies. Further, when the nature of the information consistency/ inconsistency was operationalized by way of the distribution of ratings, the results derived in the prior experiments were replicated. Thus, our central results (the moderating role of dialecticism, the dissociation between felt ambivalence and discomfort for high dialectics, as well as the moderated mediation mechanism) possess considerable generalizability, applying well across both specific and abstract levels of product attributes, and functioning independently of the ways in which information inconsistency is exhibited.

\section{General discussion}

\section{Results and contributions}

Though the construct of dialecticism is clearly important, its effect on consumers' processing of brand-related messages has not previously been studied in depth (Wyer \& Hong, 2010). We examined here the moderating effects of dialectical thinking not only on the processing of "mixed-information" messages about brand strengths and weaknesses (similar to those commonly found today on brand review websites) but also on purely negative and purely positive messages. Our series of studies makes the following five contributions.

First, we significantly broadened our theoretical understanding of the mechanisms by which dialecticism influences the processing of product information messages. We argued and showed that high dialectics should be more active searchers for "the other side of things" (opposite in valence to that which is seen) more when the incoming information is negative than when it is positive (or obviously mixed). We then used this motivational component of dialecticism to show differences in the degree of ACR generation that consumers report for presented information-so that even presented univalent information can create felt ambivalence (consistent with the arguments and evidence of Priester et al., 2007 and Priester \& Petty, 2001).

Empirically, we established the novel result that for high dialectics, univalent negative product information produces the same strong degree of felt ambivalence, and even greater discomfort, than is observed in mixed-information messages. For low dialectics, however, it was mixed information that produced the highest levels of felt ambivalence and discomfort, higher than either positive or negative messages. Our process data (tests of mediation and moderated mediation in Study 2) show clearly, through our measure of ACRs, that it is these-and not message trustworthiness, or consumer optimism or task involvement-that underlie the observed differences in levels of felt ambivalence and discomfort across dialecticism levels.

Second, we showed a very important asymmetry in the effects of univalent messages that contain positive-only versus negative-only messages: for high dialectics, negative information evoked more felt ambivalence and discomfort than positive. This asymmetry did not obtain for lower dialecticism consumers, who formed similar (and relatively less) felt ambivalence and discomfort in response to messages containing positive and negative information. These findings of an asymmetric effect, replicated in our multiple studies, are a novel contribution to dialecticism research. Dialectical thinking is widely presumed to be responsible for providing a balanced view or endorsement of positive and negative attributes of a focal object. As a result, it is easy to expect that higher dialecticism consumers should feel high attitudinal ambivalence and discomfort equally for positive or negative information messages. Our results show otherwise.

While this finding is new to the literature, our theoretical development above provides a strong rationale for why this asymmetry should arise. We argue, using several streams of prior research, that high (but not low) dialectical consumers should experience more ACR generation when faced with negative than with positive information. This should lead to a greater co-occurrence of positive and negative information and affect, therefore greater felt ambivalence and discomfort, for high dialectics receiving negative-only information. Our tests using the reported level of generated ACRs support our theorizing. We thus add to the important literature on negative information by showing that the previously shown greater influence of negative over positive information does not obtain in all circumstances. That is, while prior research shows negative information always being utilized more, and thus more influential (e.g. Skowronski \& Carlston, 1989), we show that high versus low dialectics respond in a different manner to information valence. While low dialectics indeed process negative information as unambiguously negative, high dialectics instead (because of the greater number of positive ACRs they self-generate) perceive that the true state of affairs is likely to be not as negative as stated in the presented information. Thus, high dialectics should show less of the overweighting of negative over positive information than the literature has previously documented. Negative brand information (including that on brand transgressions, or in online reviews) should thus have less impact on high dialectics. Future research could test such possibilities explicitly.

Third, though our examination of low dialectics consumers revealed parallel effects across the two dependent variables of felt ambivalence and discomfort, our study shows - for the first time - that for high dialectics, these effects are not parallel. The different effects on felt ambivalence compared to discomfort that were observed for high dialectics serve as novel empirical evidence for the process view that while high dialectics do perceive that mixed-information messages make it harder to form judgments (thus creating felt ambivalence), such messages do not create high discomfort in them. Instead, it is negative information that creates both high felt ambivalence and high discomfort for them.

Fourth, our series of experiments provided support for our hypothesized outcomes by using multiple complementary operationalizations. Experiments 1 and 2 used common but different product settings (MP3 players and Pad tablets) and exposed respondents to positive, negative, and mixed attribute- 
level information (only positive and negative in study 2 ), and to primed (manipulated) dialecticism. Experiment 3 used summary instead of the specific attribute information that was the focus in Experiments 1 and 2 and communicated information inconsistency via the distribution of customer responses to a summary index of customer satisfaction. Our results were replicated across the three studies. Our central conclusions (the moderation by dialecticism, the dissociation between felt ambivalence and discomfort for high dialectics, and the moderated mediation mechanism) can therefore be generalized to multiple real-world formats of product information presentation.

Finally, our novel method of manipulating (priming) dialecticism on a within-culture basis-consistent with the currently favored "situated cognition" perspective (e.g., Oyserman \& Sorensen, 2009) — went well beyond the methods used in previous studies (Spencer-Rodgers et al., 2004; Williams \& Aaker, 2002) and should itself be of value to future academic research. Prior studies typically assumed, or simply measured, betweenculture differences in dialecticism and similar constructs (Aaker \& Sengupta, 2000; Williams \& Aaker, 2002). In prior research manipulating dialecticism (Spencer-Rodgers et al., 2004; SpencerRodgers et al., 2010), differences were only examined between a group of dialectics and a control group, leading to results that are more ambiguous than those we provide through a manipulated high versus low dialectical comparison. We do acknowledge that our manipulation used two techniques (proverb completion and paragraph-induced story recall) and could be viewed as 'heavy-handed'. While these double manipulations made the effects stronger, they do leave some uncertainty about which one of them is more crucial, and may have created higher dialecticism than occurs in daily life.

\section{Limitations and future research}

Naturally, our studies suffer from some other limitations as well, which need to be addressed in future research. These include weak alpha reliability coefficients for some scales including DSS, suggesting the need for more DSS scale development work in cross-cultural contexts, and for further analyzing its possibly multi-factor structure. ACRs, too, need more measure development: multiple rather than single scales should be used in future research. There was also arguably a confound in our last study, where the mixed-information condition differed not only in the variance of the information but also the mean ( 3 stars) which could equate to a neutral (not just mixed) message. However, the fact that higher dialecticism consumers did feel high discomfort in that condition argues against this message as being seen as merely neutral. We note also that while our studies were conducted in China since that is an appropriate location to study dialecticism-because it is an especially noteworthy aspect of Chinese culture (Nisbett et al., 2001) - the within-culture effects we found in China might well differ in other cultures, or in a comparison between China and North America. More work is also needed to see how effects might differ in other product categories (beyond the electronics we studied) and in other domains.

\section{Appendix A. Supplementary data}

Supplementary data to this article can be found online at http://dx.doi.org/10.1016/j.jcps.2015.10.003.

\section{References}

Aaker, J. L., \& Sengupta, J. (2000). Additivity versus attenuation: The role of culture in the resolution of information incongruity. Journal of Consumer Psychology, 9(2), 67-82.

Baumeister, R. F., Bratslavsky, E., Finkenauer, C., \& Vohs, K. D. (2001). Bad is stronger than good. Review of General Psychology, 5(4), 323-370.

Choi, I., \& Nisbett, R. E. (2000). Cultural psychology of surprise: Holistic theories and recognition of contradiction. Journal of Personality and Social Psychology, 79(6), 890-905.

Choi, I., Dalal, R., Kim-Prieto, C., \& Park, H. (2003). Culture and judgement of causal relevance. Journal of Personality and Social Psychology, 84(1), 46-59.

Cialdini, R. B., Trost, M. R., \& Newsom, J. T. (1995). Preference for consistency: The development of a valid measure and the discovery of surprising behavioral implications. Journal of Personality and Social Psychology, 69(2), 318-328.

Festinger, L. (1957). A theory of cognitive dissonance. Stanford university press.

Harmon-Jones, E. (2000). Cognitive dissonance and experienced negative affect: Evidence that dissonance increases experienced negative affect even in the absence of aversive consequences. Personality and Social Psychology Bulletin, 26(12), 1490-1501.

van Harreveld, F., van der Pligt, J., \& Yael, N. (2009). The agony of ambivalence and ways to resolve it: Introducing the MAID model. Personality and Social Psychology Review, 13(1), 45-61.

Hayes, A. F. (2013). An introduction to mediation, moderation, and conditional process analysis. New York: The Guilford Press.

Herr, P. M., Kardes, F. R., \& Kim, J. (1991). Effects of word-of-mouth and product-attribute information on persuasion: An accessibility-diagnosticity perspective. Journal of Consumer Research, 17(4), 454-462.

Kaplan, K. J. (1972). On the ambivalence-indifference problem in attitude theory and measurement: A suggested modification of the semantic differential technique. Psychological Bulletin, 77(5), 361-372.

Lee, A. Y., Aaker, J. L., \& Gardner, W. L. (2000). The pleasures and pains of distinct self-construals: The role of interdependence in regulatory focus. Journal of Personality and Social Psychology, 78(6), 1122-1134.

Markus, H. R., \& Kitayama, S. (1991). Culture and the self: Implications for cognition, emotion, and motivation. Psychological Review, 98(2), 224-253.

Mick, D. G., \& Fournier, S. (1998). Paradoxes of technology: Consumer cognizance, emotions, and coping strategies. Journal of Consumer Research, 25(2), 123-143.

Miyamoto, Y., Uchida, Y., \& Ellsworth, P. C. (2010). Culture and mixed emotions: Co-occurrence of positive and negative emotions in Japan and the United States. Emotion, 10(3), 404-415.

Monga, A. B., \& John, D. R. (2008). When does negative brand publicity hurt? The moderating influence of analytic versus holistic thinking. Journal of Consumer Psychology, 18(4), 320-332.

Newby-Clark, I. R., McGregor, I., \& Zanna, M. P. (2002). Thinking and caring about cognitive inconsistency: When and for whom does attitudinal ambivalence feel uncomfortable? Journal of Personality and Social Psychology, 82(2), 157-166.

Nisbett, R. E., Peng, K., Choi, I., \& Norenzayan, A. (2001). Culture and systems of thought: Holistic versus analytic cognition. Psychological Review, 108(2), 291-310.

Oyserman, D., \& Sorensen, N. (2009). Understanding cultural syndrome effects on what and how we think: A situated cognition model. In R. S. Wyer, C. -Y. Chiu, \& Y. -Y. Hong (Eds.), Understanding Culture: Theory, Research and application. New York: Psychology Press.

Peng, K., \& Nisbett, R. E. (1999). Culture, dialectics, and reasoning about contradiction. American Psychologist, 54(9), 741-754. 
Petrocelli, J. V., Tormala, Z. L., \& Rucker, D. D. (2007). Unpacking attitude certainty: Attitude clarity and attitude correctness. Journal of Personality and Social Psychology, 92(1), 30-41.

Priester, J. R., \& Petty, R. E. (1996). The gradual threshold model of ambivalence: Relating the positive and negative bases of attitudes to subjective ambivalence. Journal of Personality and Social Psychology, 71(3), 431-449.

Priester, J. R., \& Petty, R. E. (2001). Extending the bases of subjective attitudinal ambivalence: Interpersonal and intrapersonal antecedents of evaluative tension. Journal of Personality and Social Psychology, 80(1), 19-34.

Priester, J. R., Petty, R. E., \& Park, K. (2007). Whence univalent ambivalence? From the anticipation of conflicting reactions. Journal of Consumer Research, 34(1), 11-21.

Rucker, D. D., Tormala, Z. L., Petty, R. E., \& Briñol, P. (2014). Consumer conviction and commitment: An appraisal-based framework for attitude certainty. Journal of Consumer Psychology, 24(1), 119-136.

Schwarz, N., \& Bless, H. (1991). Happy and mindless, but sad and smart? The impact of affective states on analytic reasoning. In J. P. Forgas (Ed.), Emotion and social judgments (pp. 55-71). Elmsford, NY: Pergamon.
Sengupta, J., \& Johar, G. V. (2002). Effects of inconsistent attribute information on the predictive value of product attitudes: Toward a resolution of opposing perspectives. Journal of Consumer Research, 29(1), 39-56.

Skowronski, J. J., \& Carlston, D. E. (1989). Negativity and extremity biases in impression formation: A review of explanations. Psychological Bulletin, 105(1), 131-142.

Spencer-Rodgers, J., Peng, K., \& Wang, L. (2010). Dialecticism and the cooccurrence of positive and negative emotions across cultures. Journal of Cross-Cultural Psychology, 41(1), 109-115.

Spencer-Rodgers, J., Peng, K., Wang, L., \& Hou, Y. (2004). Dialectical selfesteem and East-West differences in psychological well-being. Personality and Social Psychology Bulletin, 30(11), 1416-1432.

Triandis, H. C. (1995). Individualism and collectivism. Boulder, CO: Westview.

Williams, P., \& Aaker, J. L. (2002). Can mixed emotions peacefully coexist? Journal of Consumer Research, 28(4), 636-649.

Wyer, R. S., \& Hong, J. (2010). Chinese Consumer Behavior: The Effects of Content, Process and Language. In M. H. Bond (Ed.), Oxford Handbook of Chinese Psychology (pp. 623-639) (2nd ed.). New York: Oxford University Press. 